\title{
The recent structural evolution of the SMC
}

\author{
F. Maragoudaki ${ }^{1}$, M. Kontizas ${ }^{1}$, D. H. Morgan ${ }^{2}$, E. Kontizas ${ }^{3}$, A. Dapergolas ${ }^{3}$, and E. Livanou ${ }^{1}$ \\ 1 Section of Astrophysics Astronomy \& Mechanics, Department of Physics, University of Athens, \\ 15783 Athens, Greece \\ 2 Institute for Astronomy, University of Edinburgh, Blackford Hill, Edinburgh EH9 3HJ, UK \\ 3 Institute of Astronomy and Astrophysics, National Observatory of Athens, PO Box 20048, 11810 Athens, Greece
}

Received 28 June 2001 / Accepted 15 October 2001

\begin{abstract}
We investigate the spatial distribution of stars of different ages towards the main body of the SMC, in an attempt to further understand the nature of the complex structure of the SMC. The old stellar population of the galaxy shows a rather regular and smooth distribution which is typical for a spheroidal body. On the contrary, the distribution of the younger stellar component is highly asymmetric and irregular giving evidence for the severe impact of the SMC during its close encounter with the LMC some 0.2 to 0.4 Gyr ago. In a series of isodensity contour maps of stars within selected ages, the recent structural evolution of the SMC is presented.
\end{abstract}

Key words. galaxies: Magellanic Clouds - galaxies: interactions - galaxies: evolution - galaxies: structure

\section{Introduction}

The Magellanic Clouds are an ideal laboratory for studing the consequences of galaxy interactions on the formation and evolution of stellar populations. It is believed that they have been bound to the Galaxy for at least the last 10 Gyr (Murai \& Fujimoto 1980; Fujimoto \& Murai 1984), during which time significant interactions between the LMC and the SMC as well as between the Clouds and the Galaxy have led to structural and kinematical peculiarities (Westerlund 1997). In particular, the structure of the SMC is considered to have been severely affected by the previous SMC perigalactic passage which coincided with a close encounter between the Clouds $\sim 1.5 \mathrm{Gyr}$ ago, and also by the more recent close encounter between the Clouds some 0.2 to 0.4 Gyr ago (Gardiner \& Noguchi 1996; Kunkel et al. 2000). Consequently, various dynamical models and simulations have been constructed to try to describe the mechanisms dominating these interactions.

The SMC shows an asymmetric appearance with an irregular Bar and an eastern extension, while it is highly stretched along the line of sight; the Wing and the northeastern part of the Bar are closer than the southwestern regions (Mathewson et al. 1986, 1988; Hatzidimitriou \& Hawkins 1989; Hatzidimitriou et al. 1993). Recently, Zaritsky et al. (2000) confirmed that this asymmetric structure is almost exclusively due to young mainsequence stars, the older stellar population showing an

Send offprint requests to: F. Maragoudaki,

e-mail: fmarag@cc.uoa.gr extremely regular distribution. The same conclusions were drawn by Cioni et al. (2000) from the DENIS near-infrared survey.

Even so, the structural evolution of the SMC is still obscure. There remain the questions of whether the socalled "Bar" is simply the projection effect of a severely damaged galaxy along the line of sight or a real dynamical structure, and, if so, what could have caused it?

We have begun an investigation in order to further understand the structure of the SMC. As well as finding a regular distribution for the older stellar component and an asymmetric appearance for the younger stars, we show for the first time the evolution of the perturbations developed by the LMC-SMC interactions and give a possible explanation for the absence of an SMC rotational velocity.

\section{The data}

In order to study the large-scale properties of nearby galaxies such as the SMC, we need wide-field observational materal. So, for this work we used direct photographic plates taken with the $1.2 \mathrm{~m}$ UK Schmidt Telescope in the $U, V$ and $I$ wavebands. The plates cover an area of $6^{\circ} \times 6^{\circ}$ in the plane of the sky centred on $1^{\mathrm{h}} 06^{\mathrm{m}},-75.0^{\circ}$ (B1950). (This plate centre is ESO/SERC Survey Field 29 which is offset somewhat from the main body of the SMC.) The $U$ plate is ideal for showing the young blue stars of the upper main sequence and the $I$ plate provides a good picture of the old stellar component. The $V$ plate is, of course intermediate between these showing both main-sequence stars 


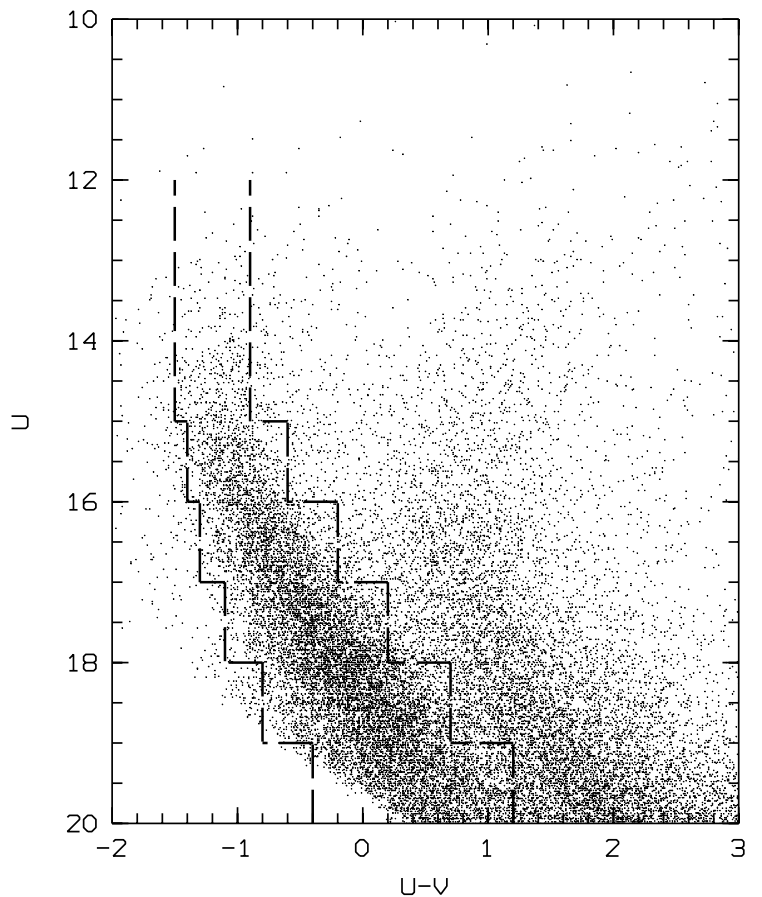

Fig. 1. a) The $(U, U-V)$ colour-magnitude diagram of stars detected in both $U$ and $V$. There is a clear separation between the main sequence stars and the other stellar populations. (Only one tenth of the paired stars are plotted here.)

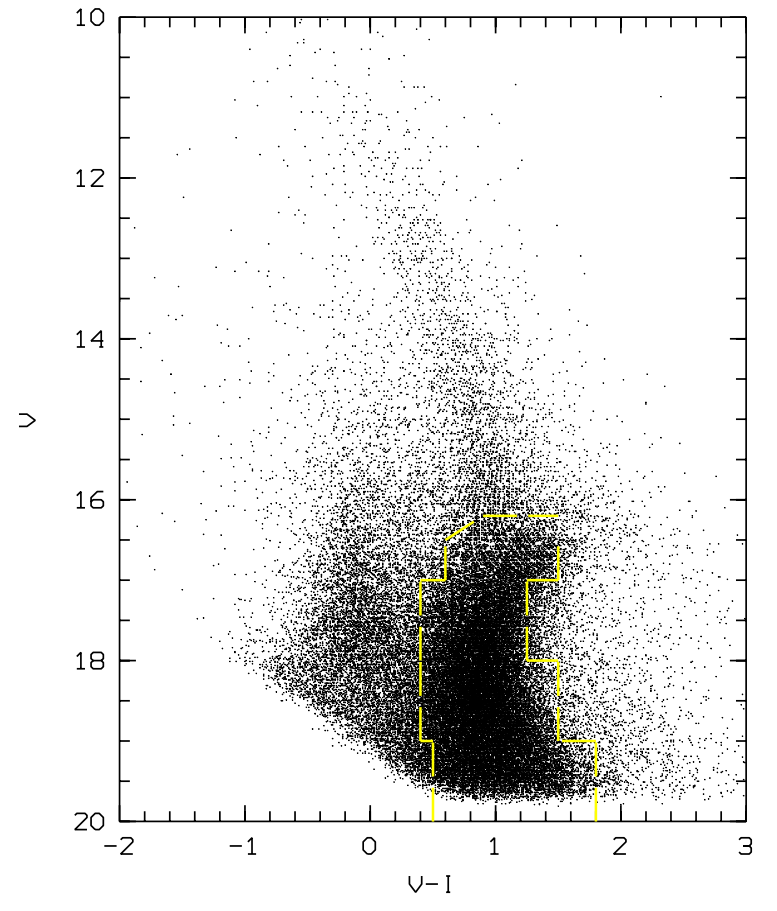

Fig. 1. b) The $(V, V-I)$ colour-magnitude diagram of stars detected in both $V$ and $I$. There is a clear separation between the giant stars and the other stellar populations. (Only one tenth of the paired stars are plotted here.)

relatively low stellar surface densities. In the crowded parts they rise to $6 \%, 21 \%$ and $47 \%$ respectively.

The $(U, U-V)$ and $(V, V-I)$ colour magnitude diagrams are shown in Fig. 1. We have used these CMDs to select different stellar populations. The areas occupied by the main sequence and the red giants/supergiants are indicated by the lines drawn in Figs. 1a and 1b respectively. Figures 1a,b show clearly the incompleteness in the $U-V$ paired data fainter than $U=18.5$ mag due to the cutoff of the $V$ plate.

We then divided the stellar catalogues into subcatalogues according to the apparent magnitude of the stars and population type (colour index) as marked in Figs. 1 and $1 \mathrm{~b}$. We assigned an age to each magnitude "slice" from the empirical CMD of main sequence stars (Zombeck 1990) as given by the $U$ magnitude after allowing for the distance modulous of the SMC ( $m-M=19.0$ according to Westerlund 1997). Using theoretical isochrones with various metallicities, we derived a range of ages which expresses the error given for each magnitude slice's age. The slice corresponding to the red giants was selected to represent stars older than 2 Gyr (Zombeck 1990).

For each selected "magnitude-colour index slice", star counts were made using a pixel size of $\sim 26 \times 26 \mathrm{pc}^{2}$. The mean background density and its rms scatter $-\sigma$ - were then determined in regions well away from the main body of the SMC. Next, the star count data were smoothed by replacing the count in each pixel by the average count in the surrounding box of $9 \times 9$ pixels, and finally, isodensity contour maps of the whole area were produced. 

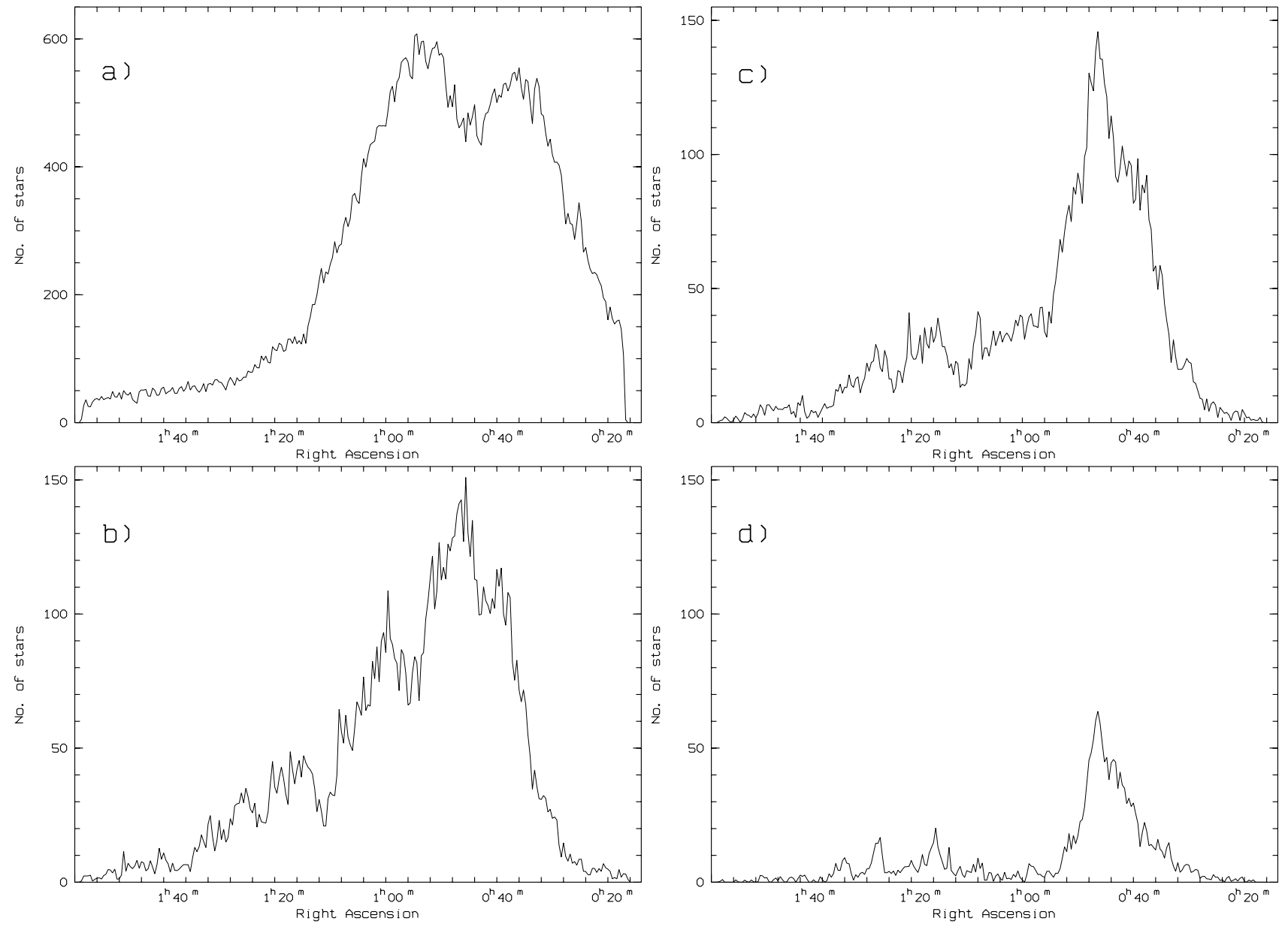

Fig. 2. Stellar density profiles of an east-west cross section around $-73.2^{\circ}$. a) illustrates the giants of the isocontour map Fig. 3 . b) -d) illustrate main sequence stars of "magnitude-color index slices" corresponding to the isocontour maps of Figs. 5 , 6 and 8 respectively.

For the red giants, the first contour level was set at $3 \sigma$ above the mean background density and the contour step was $2 \sigma$. For the main sequence stars the star count images are almost void of background stars. Therefore, the first contour was set at an initial realistic value (many times the background). In this way, we were able to investigate the spatial distribution of stars of different ages. The resulting maps are shown in Figs. 3 and 4-9 for the old giants and the various "slices" of the blue main sequence stars respectively. The coordinates are J2000. The background levels for each slice are better illustrated in Figs. 2a-d which show histograms of an east-west crosssection of $\sim 0.5^{\circ}$ width around Dec $\sim-73.2^{\circ}$. Figures $2 \mathrm{a}-$ $\mathrm{d}$ correspond to the isocontour maps of Figs. 3, 5, 6, 8 respectively.

\section{Discussion}

A first glance at the contour maps shows how stellar age changes the spatial appearance of the SMC. It should be noted that the appearance of the SMC north of Dec $\sim$ $-72^{\circ}$ is not real but is due to the proximity of the plate edge.
Figure 3 shows the old stellar population to be rather smooth and regular, leading us to describe the SMC as a spheroidal system. (The central region of the SMC in Fig. 3 was masked because the high concentration of bright red stars distorts the very faint population.) The spheroidal nature of the system can also be seen in the density profile shown in Fig. 2a. It is consistent with the distribution of other old stellar components, such as the carbon stars (Morgan \& Hatzidimitriou 1995) and the red giants (Zaritsky et al. 2000). Nor is there any difference between our data and the SMC morphology in $I$ as given by Cioni et al. (2000) for AGB and RGB stars down to their detection limit of $I \sim 16.5$. However, our data reach much fainter magnitudes $(I \sim 19.5)$ with a very good completeness factor. Although these magnitudes have been derived from an extrapolation of the calibration curve and consequently have a relatively large uncertainty, they are good enough for us to be sure that Fig. 3 represents a very old SMC stellar population which show no signs of tidal or hydrodynamical distortion.

Figures 4-9 which show the spatial distribution of the blue stars, illustrate the "transition" of an initially spheroidal body to a highly asymmetric and irregular 


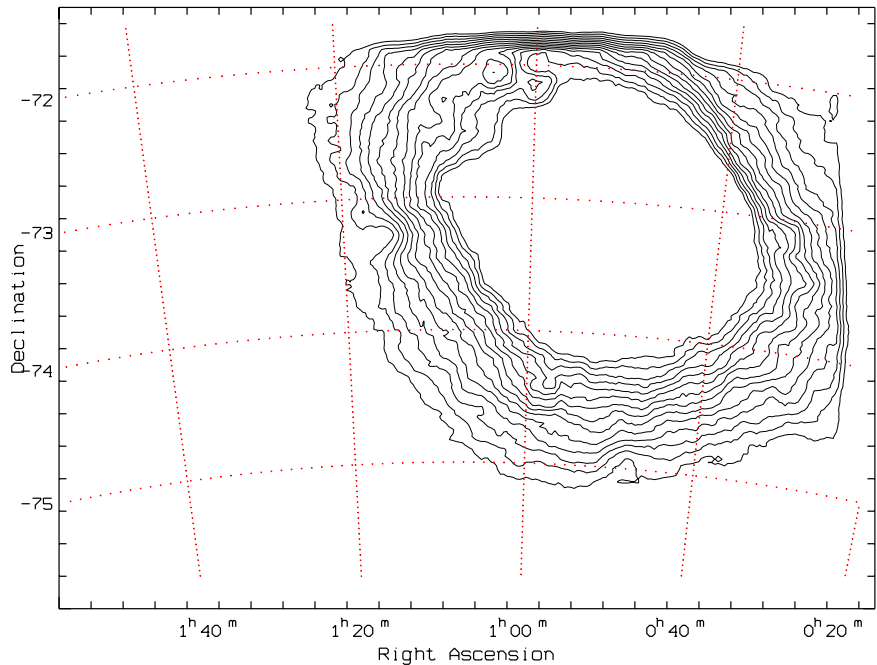

Fig. 3. Isodensity contour map of SMC giants $(17<V<20$ and $\sim 0.4<V-I<\sim 1.8)$. The central region was masked because the high concentration of bright red stars distorts the very faint population. The distribution of the old SMC population (older than 2 Gyr) is smooth and regular with an elongation towards the direction of the LMC.

system. We can now see many of the common features of the SMC that were absent from Fig. 3, such as the Wing, the so-called "SMC Bar" and the Tail. Although Fig. 4 was produced from a "slice" which suffers from significantly greater incompleteness than the others, it is included because it shows that both the Wing and the Tail are already evident $\left(\sim 3.4-4 \times 10^{8} \mathrm{yr}\right)$. The "Bar" is most prominent in Figs. 8 and 9.

This series of the isodensity maps (Figs. 5-9) provides strong evidence for a subsequent fragmentation of "star filaments" in the area around $1^{\mathrm{h}} 10^{\mathrm{m}},-73^{\circ} 10^{\prime}$, followed by a concentration of star formation in the south west part of the SMC and in the Wing. It seems that the new generations of stars preferentially appear along a Northeast-Southwest direction, giving rise to the so-called Bar. Another interesting feature seen in these maps is a rather circular concentration of stars, a "shell", centred on $\sim 1^{\mathrm{h}} 05^{\mathrm{m}},-72^{\circ} 30^{\prime}$, also mentioned by Zaritsky et al. (2000).

These results are also evident in the cross-sections of Figs. 2b-d which show the absolute star counts. For example, the counts between positions $\sim 1^{\mathrm{h}} 08^{\mathrm{m}}$ and $1^{\mathrm{h}} 52^{\mathrm{m}}$ are approximately one twentieth of the peak counts associated with the Bar (at position $\sim 0^{\mathrm{h}} 40^{\mathrm{m}}$ ) in the bright "slices", but rise by a factor of $\sim 13$ to around two thirds of the peak counts for the faint slice of Fig. 2d. Whilst, we expect the greatest loss of image detection to be in the Bar region, this differential loss cannot explain the observed thirteen-fold change in the count ratio.

The asymmetric appearance of the young population of the SMC as seen here and by Zaritsky et al. (2000) and Cioni et al. (2000) is also consistent with the patterns of the associations, clusters and HII regions (Bica \& Schmitt 1995). The distribution of the HI in the SMC

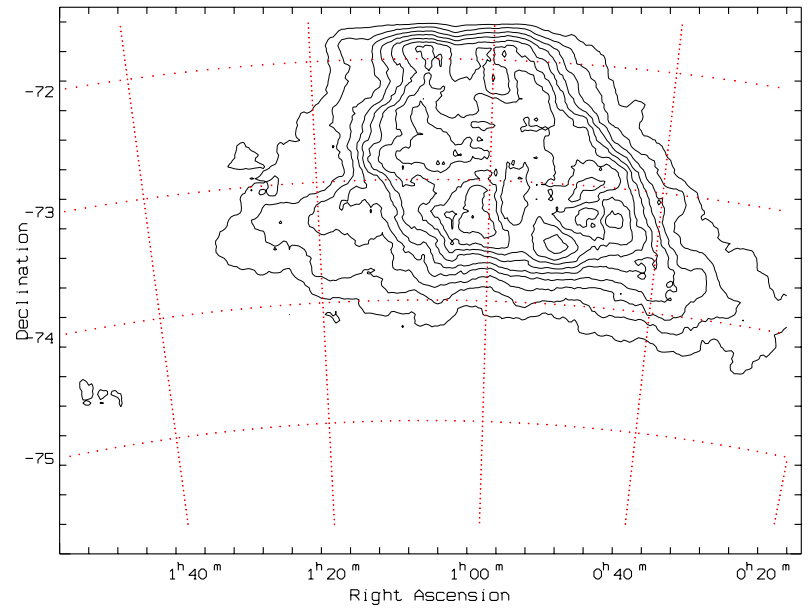

Fig. 4. Isodensity contour map of main sequence stars with $19<U<20$ and $-0.4<U-V<1.2$, corresponding approximately to age $3.4-4 \times 10^{8} \mathrm{yr}\left( \pm 0.5 \times 10^{8} \mathrm{yr}\right)$. The Wing and the Tail are already evident.

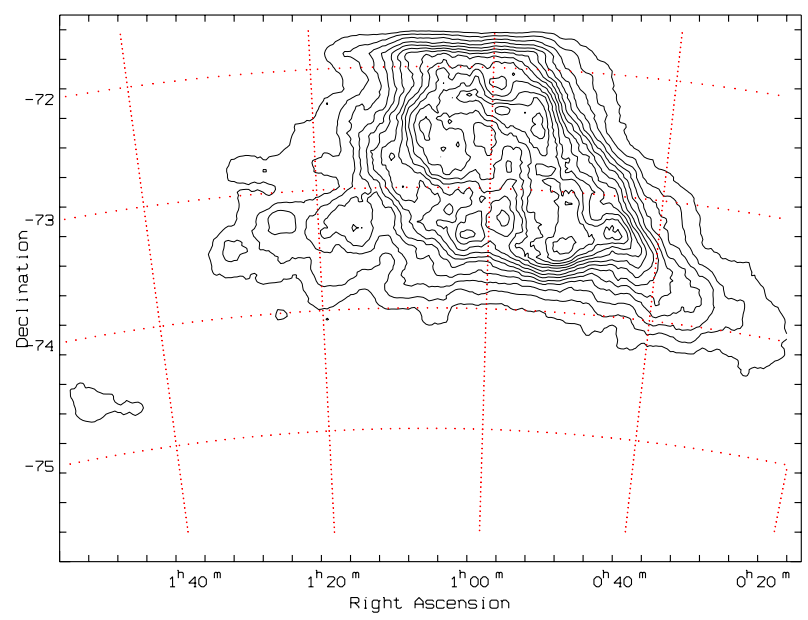

Fig. 5. Isodensity contour map of main sequence stars with $18<U<19$ and $-0.8<U-V<0.7$, corresponding to age $1.7-3.4 \times 10^{8}$ yr $\left( \pm 0.5 \times 10^{8} \mathrm{yr}\right)$. The fragmentation of star filaments in the area around $1^{\mathrm{h}} 10^{\prime},-73^{\circ} 10^{\prime}$ has begun to appear.

(see Fig. 3 in Stanimirovic et al. 1999) is dominated by a central concentration associated with the optical Bar and an eastern Wing, with the Bar and the Wing being connected by a "bridge"; this pattern is also seen in Figs. 6-8. The fact that the old population has no signs of any of these features means that it has remained undisturbed by the recent LMC-SMC encounter. Therefore, forces other than just tidal must have played a key role in the dynamics of the HI and the evolution of the SMC.

According to the numerical simulations of Gardiner \& Noguchi (1996), many of the observed features of the SMC are the result of the close encounter with the LMC 0.2-0.4 Gyr ago (or 0.3-0.4 Gyr - Kunkel et al. 2000). Figure 4 clearly shows that at the time of $3.4-4 \times 10^{8} \mathrm{yr}$ the Wing and the Tail were already prominent features in the structure of the SMC. 


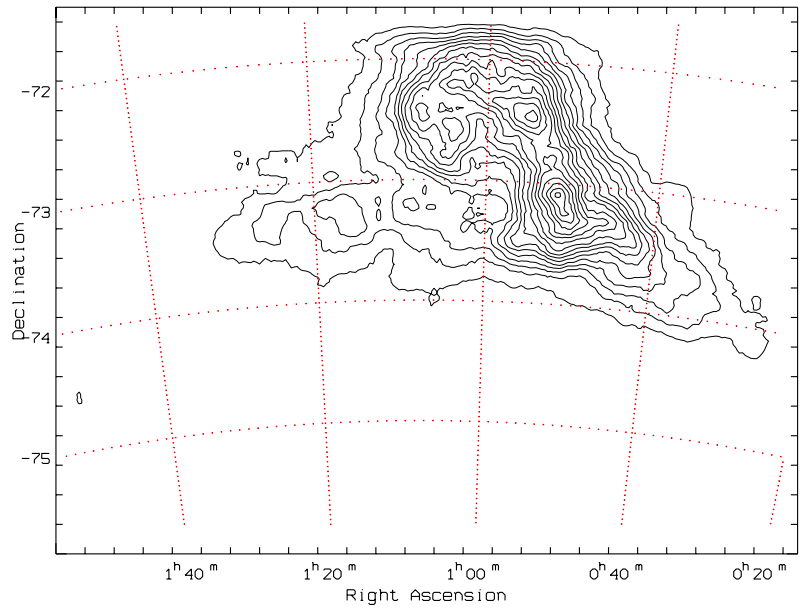

Fig. 6. Isodensity contour map of main sequence stars with $17<U<18$ and $-1.1<U-V<0.2$, corresponding to age $3 \times 10^{7} \mathrm{yr}-1.7 \times 10^{8} \mathrm{yr}\left( \pm 10^{7} \mathrm{yr}\right)$. The fragmentation mentioned above is followed by the formation of a "shell" in the northern SMC and a higher concentration of stars in the southwest region of the SMC.

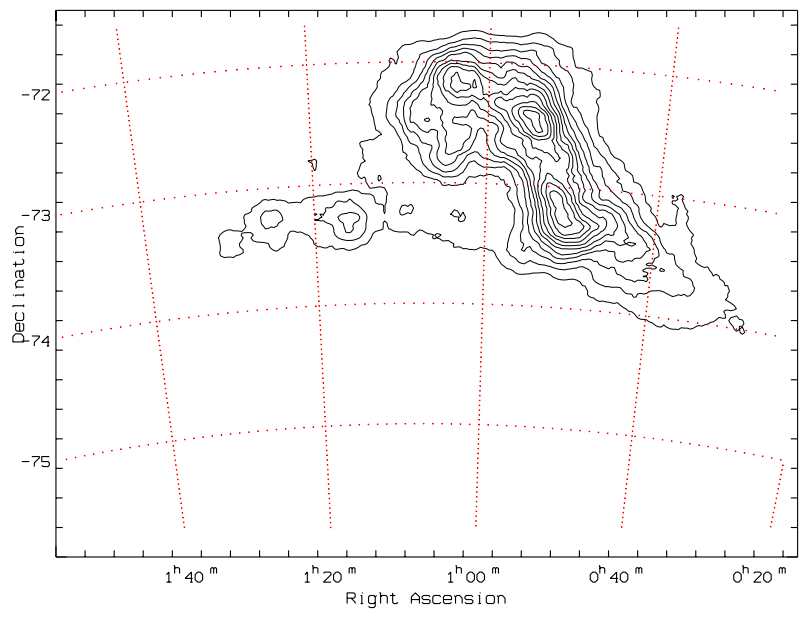

Fig. 7. Isodensity contour map of main sequence stars with $16<U<17$ and $-1.3<U-V<-0.2$, corresponding to age $(1.2-3) \times 10^{7} \mathrm{yr}$. The "Bar" of the SMC is now more marked.

Comparing the isodensity maps of the SMC with those presented by Maragoudaki et al. (1998) for the LMC, it becomes clear that in each galaxy the distribution of stellar populations tends to become more regular and well ordered as its age increases. It is also evident that, at the time of close approach, the LMC Bar was already a well defined structure, in the contrast with the SMC. The interaction of the two galaxies also affected the structure of the LMC by triggering extended bursts of star formation, though in a less dramatic way than in the case of the SMC.

If we accept that we are witnessing the formation of a Bar in the SMC, then we have to explain the lack of angular momentum, since no obvious angular velocity has been so far observed (Kunkel et al. 2000). According to Gerin et al. (1990), special conditions of the geometry of the two interacting galaxies can lead to a decrease in the angular momentum of the one of the interacting bodies. However,

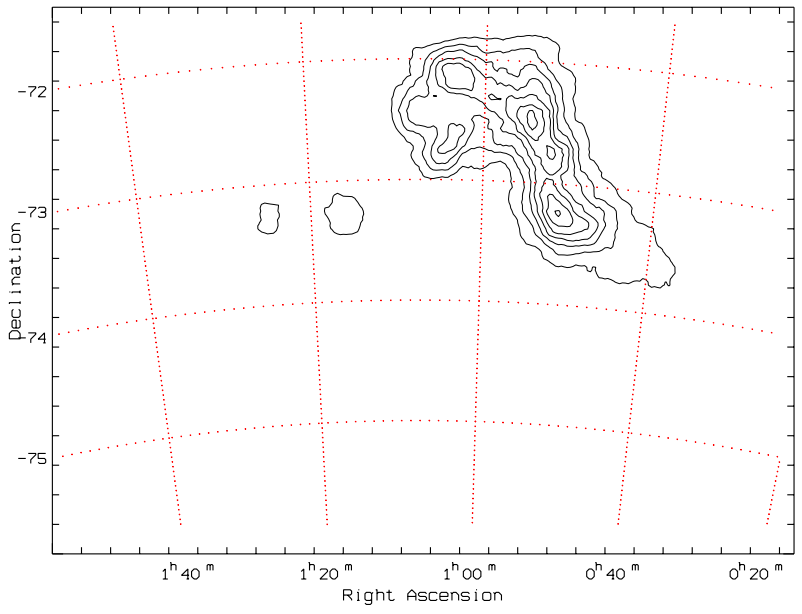

Fig. 8. Isodensity contour map of main sequence stars with $15<U<16$ and $-1.4<U-V<-0.6$, corresponding to age $8 \times 10^{6}-1.2 \times 10^{7} \mathrm{yr}$.

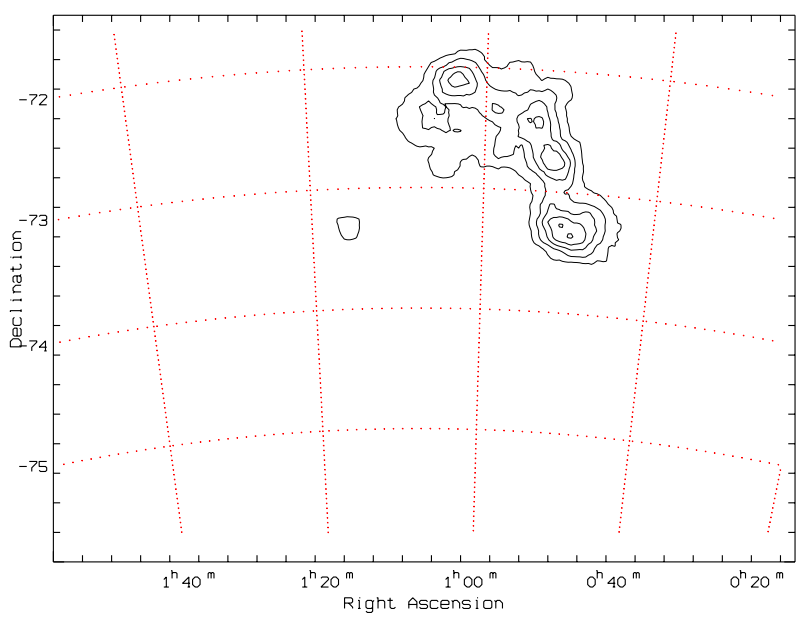

Fig. 9. Isodensity contour map of main sequence stars with $U<15$ and $-1.5<U-V<-0.8$, corresponding to age younger than $8 \times 10^{6} \mathrm{yr}$. The youngest SMC stellar population is now concentrated in the "Bar" and the Wing.

it is not certain that this feature is a "genuine bar", and not a temporary extended star burst region. In any case, additional velocity observations are needed to clarify the exact kinematical properties of the SMC. Improved numerical models also need to take into consideration hydrodynamic effects as well as tidal forces, to reproduce all the characteristics of the observed SMC structure through time.

Acknowledgements. The authors would like to thank the referee D. Bomans for his constructive suggestions and critical comments. They would also like to express their thanks to the $1.2 \mathrm{~m}$ UK Schmidt Telescope Unit for the loan of the plates and the SuperCOSMOS for the digitization of them. Financial support for this project was provided by the British Council and the General Secretariat of Research and Technology. 


\section{References}

Bica, E. L. D., \& Schmitt, H. R. 1995, AJS, 101, 41

Butler, C. J. 1972, Dunsink Obs. Publs., 1(6), 133

Cioni, M.-R. L., Habing, H. J., \& Israel, F. P. 2000, A\&A, 358, L9

Fujimoto, M., \& Murai, T. 1984, in IAU Symp. 108, ed. S. van den Bergh, \& K. S. de Boer (Reidel, Dordrecht), 115

Gardiner, L. T., \& Noguchi, M. 1996, MNRAS, 278, 191

Gerin, M., Combes, F., \& Athanassoula, E. 1990, A\&A, 230, 37

Hatzidimitriou, D., Cannon, R. D., \& Hawkins, M. R. S. 1993, MNRAS, 261, 873

Hatzidimitriou, D., \& Hawkins, M. R. S. 1989, MNRAS, 241, 667

Kunkel, W. E., Demers, S., \& Irwin, M. J. 2000, AJ, 119, 2789

Maragoudaki, F., Kontizas, M., Kontizas, E., Dapergolas, A., \& Morgan, D. H. 1998, A\&A, 338, L29
Mathewson, D. S., Ford, V. L., \& Visvanathan, N. 1988, ApJ, 333,617

Mathewson, D. S., Ford, V. L., \& Visvanathan, N. 1986, ApJ, 301,664

Morgan, D. H., \& Hatzidimitriou, D. 1995, A\&AS 113, 539

Murai, T., \& Fujimoto, M. 1980, PASJ, 32, 581

Postman, M., Bucciarelli, B., Sturch, C., et al. 1997, in New Horizons from Multi-wavelength Sky Surveys, ed. B. J. McLean et al., Proc IAU Symp., 179, 379

Stanimirovic, S., Staveley-Smith, L., Dickey, J. M., Sault, R. J., \& Snowden, S. L. 1999, MNRAS, 302, 417

Westerlund, B. E. 1997, The Magellanic Clouds, Camb. Astr. Ser., 29

Zaritsky, D., Harris, J., Grebel, E. K., \& Thompson, I. B. 2000, ApJ, 534, L53

Zombeck, M. V. 1990, Handbook of Astronomy \& Astrophysics (Camb. Univ. Pres.) 